\title{
Assessing the chemical stability and cytotoxicity of electrodeposited magnetic mesoporous Fe-Pt films for biomedical applications.
}

Albert Serrà a,b, *, David Limón ${ }^{b, c}$, Natalia Díaz-Garrido, ${ }^{d}$ Lluïsa Pérez-García ${ }^{b, c}$, and

Elvira Gómez a,b,*

a Thin Films and Nanostructures Group, Department of Materials Science and Physical

Chemistry, Facultat de Química, Universitat de Barcelona, Martí i Franquès, 1, E-

08028, Barcelona, Catalonia, Spain.

${ }^{b}$ Institute of Nanoscience and Nanotechnology (IN $\left.{ }^{2} \mathrm{UB}\right)$, Universitat de Barcelona, Barcelona, Catalonia, Spain.

${ }^{c}$ Departament de Farmacologia, Toxicologia i Química Terapèutica, Facultat de

Farmàcia i Ciències de l'Alimentació, Universitat de Barcelona, Avda. Joan XXIII 27-

31, E-08028 Barcelona, Catalonia, Spain.

${ }^{\mathrm{d}}$ Departament de Bioquímica i Fisiologia, Facultat de Farmàcia i Ciències de

l'Alimentació, Universitat de Barcelona, Avda. Joan XXIII 27-31, E-08028 Barcelona,

Catalonia, Spain. 
Corresponding author: a.serra@ub.edu (A.S.); e.gomez@ub.edu (E.G.)

\section{Contents}

Cytotoxicity of $\mathrm{Fe}-\mathrm{Pt}$ mesoporous films 
Cytotoxicity of Fe-Pt mesoporous films

\begin{tabular}{|l|c|c|c|c|c|}
\hline & Replicate 1 & Replicate 2 & Replicate 3 & Mean & SD \\
\hline CONTROL & $99.7 \%$ & $96.2 \%$ & $104.1 \%$ & $100.0 \%$ & $4.0 \%$ \\
\hline Control Si/Ti/Au & $104.9 \%$ & $98.4 \%$ & $93.1 \%$ & $98.8 \%$ & $5.9 \%$ \\
\hline FePt -900mV & $107.6 \%$ & $102.4 \%$ & $114.6 \%$ & $108.2 \%$ & $6.1 \%$ \\
\hline FePt - $1,000 \mathrm{mV}$ & $113.1 \%$ & $119.8 \%$ & $118.7 \%$ & $117.2 \%$ & $3.6 \%$ \\
\hline FePt -1,100 mV & $104.3 \%$ & $114.3 \%$ & $105.1 \%$ & $107.9 \%$ & $5.5 \%$ \\
\hline
\end{tabular}

Table S1. Cell viability of $\mathrm{HaCaT}$ cells after 48 -h incubation in presence of $\mathrm{Si} / \mathrm{Ti} / \mathrm{Au}$

substrates with Fe-Pt films deposited at $-900,-1,000$, and $-1,100 \mathrm{mV}$, or without Fe-Pt,

relative to untreated cells (control).

\begin{tabular}{|c|c|c|}
\hline P value & \multicolumn{2}{|l|}{0.4060} \\
\hline Exact or approximate $\mathrm{P}$ value? & \multicolumn{2}{|c|}{ Gaussian Approximation } \\
\hline P value summary & \multicolumn{2}{|l|}{ ns } \\
\hline Do the medians vary signif. $(\mathrm{P}<0.05)$ & \multicolumn{2}{|l|}{ No } \\
\hline Number of groups & \multicolumn{2}{|l|}{5} \\
\hline Kruskal-Wallis statistic & \multicolumn{2}{|l|}{4.000} \\
\hline Dunn's Multiple Comparison Test & $\begin{array}{l}\text { Difference in } \\
\text { rank sum }\end{array}$ & $\begin{array}{c}\text { Significant } \\
(\mathrm{P}<0.05)\end{array}$ \\
\hline CONTROL vs Si/Ti/Au & 1.000 & No \\
\hline CONTROL vs Si/Ti/Au - FePt -900 mV & -2.000 & No \\
\hline CONTROL vs Si/Ti/Au - FePt $-1,000 \mathrm{mV}$ & -3.000 & No \\
\hline CONTROL vs Si/Ti/Au - FePt -1,100 mV & -1.000 & No \\
\hline $\mathrm{Si} / \mathrm{Ti} / \mathrm{Au}$ vs Si/Ti/Au - FePt $-900 \mathrm{mV}$ & -3.000 & No \\
\hline $\mathrm{Si} / \mathrm{Ti} / \mathrm{Au}$ vs $\mathrm{Si} / \mathrm{Ti} / \mathrm{Au}-\mathrm{FePt}-1,000 \mathrm{mV}$ & -4.000 & No \\
\hline $\mathrm{Si} / \mathrm{Ti} / \mathrm{Au}$ vs Si/Ti/Au - FePt $-1,100 \mathrm{mV}$ & -2.000 & No \\
\hline $\mathrm{Si} / \mathrm{Ti} / \mathrm{Au}-\mathrm{FePt}-900 \mathrm{mV}$ vs Si/Ti/Au - FePt $-1,000 \mathrm{mV}$ & -1.000 & No \\
\hline $\mathrm{Si} / \mathrm{Ti} / \mathrm{Au}$ - FePt -900 mV vs Si/Ti/Au - FePt -1,100 mV & 1.000 & No \\
\hline $\mathrm{Si} / \mathrm{Ti} / \mathrm{Au}-\mathrm{FePt}-1,000 \mathrm{mV}$ vs Si/Ti/Au - FePt $-1,100 \mathrm{mV}$ & 2.000 & No \\
\hline
\end{tabular}

Table S2. Kruskal-Wallis test with Dunn's Multiple Comparison Test of relative cell

viability of $\mathrm{HaCaT}$ cells after 48 -h incubation in presence of $\mathrm{Si} / \mathrm{Ti} / \mathrm{Au}$ substrates with or without Fe-Pt. 
